\title{
Combined momentum collimation studies in a high-intensity rapid cycling proton synchrotron
}

\author{
Jing-Yu Tang, ${ }^{1,2, *}$ Jin-Fang Chen, ${ }^{1}$ and Ye Zou ${ }^{2,1}$ \\ ${ }^{1}$ Institute of High Energy Physics, CAS, Yuquan Road 19B, Beijing 100049, People's Republic of China \\ ${ }^{2}$ National Synchrotron Radiation Laboratory, University of Science and Technology of China, \\ Hefei, Anhui 230029, People's Republic of China \\ (Received 30 January 2011; published 16 May 2011)
}

\begin{abstract}
Momentum collimation in a high-intensity rapid cycling synchrotron (RCS) is a very important issue. Based on the two-stage collimation principle, a combined momentum collimation method is proposed and studied in detail here. The method makes use of the combination of secondary collimators in both the longitudinal and transverse planes. The primary collimator is placed at a high-dispersion location of an arc, and the longitudinal and transverse secondary collimators are in the same arc and in the adjacent downstream dispersion-free long straight section, respectively. The particles with positive momentum deviations will be scattered and degraded by a carbon scraper and then collected mainly by the transverse collimators, whereas the particles with negative momentum deviations will be scattered by a tantalum scraper and mainly collected by the longitudinal secondary collimators. This is to benefit from the different effects of protons passing through a high atomic number material and a low atomic number material, as the former produces relatively more scattering than the latter for the same energy loss. The studies also reveal that momentum collimation is strongly dependent on the transverse beam correlation that comes from the injection painting. The relevant requirements on the lattice design are also discussed, especially for compact rings. The multiparticle simulations using both TURTLE and ORBIT codes are presented to show the physical images of the collimation method, which was carried out with the input of the RCS of China Spallation Neutron Source.
\end{abstract}

DOI: 10.1103/PhysRevSTAB.14.050103

\section{INTRODUCTION}

Momentum collimation in a high-intensity proton rapid cycling synchrotron (RCS) is a very important issue, because large beam losses during rf capture and acceleration stages contribute a large portion to the total beam loss. The relevant beam losses are due to the fast acceleration and the longitudinal space charge in such an accelerator. Even with a chopped beam injection, there are also some longitudinal halo particles between bunches that will result in beam loss. Another beam loss mechanism in the longitudinal plane is related to the particles that lose large energy in the transverse collimators but survive from there. The uncontrolled beam loss is very important for hands-on maintenance and the safety of accelerator devices. Even more, the lattice design usually has to be optimized to make the momentum collimation efficient.

The two-stage collimation method is considered to be more efficient than the single-stage collimation method in a synchrotron where the impact depth for the collimation of halo particles is usually very small, especially when the beam energy is in hundreds of $\mathrm{MeV}$ or higher. After having

\footnotetext{
*Corresponding author. tangjy@ihep.ac.cn

Published by the American Physical Society under the terms of the Creative Commons Attribution 3.0 License. Further distribution of this work must maintain attribution to the author(s) and the published article's title, journal citation, and DOI.
}

PACS numbers: 41.75.-i, 29.20.dk, 29.27.Eg, 41.85.Si

studied the collimation methods used in other highintensity synchrotrons [1-3], we found that it is difficult to design a momentum collimation system with high collimation efficiency. The collimation efficiency is defined as the ratio of collimator absorbed particles to total lost particles. The main reason is that there is usually no sufficient space in arcs, as arc regions are usually designed to be compact with little straight sections for the reason that many devices are not suitable to be placed there due to large dispersion. A new momentum collimation method-so-called combined momentum collimation method-is proposed here, which makes use of two-stage collimation in both the longitudinal and the transverse phase planes. Numerical simulations using TURTLE [4] and ORBIT [5] codes have been carried out to check the validity of the method. It turns out that this method gives high collimation efficiency for medium-energy synchrotrons. The studies have also shown two interesting effects: one is that the momentum collimation is strongly dependent on the transverse beam correlation that comes from the injection painting in the phase space; the other is that the material for the primary momentum collimator plays an important role in the collimation method.

Although the method is general and applicable for all RCS types of accelerators, the studies presented here are mainly based on the RCS parameters of China Spallation Neutron Source (CSNS) [6,7]. The design features and main parameters of the RCS are given in the Appendix. The collimation efficiencies for different acceleration 
scenarios have also been calculated to show the generality of the method.

\section{STUDIES ON MOMENTUM COLLIMATION METHODS}

\section{A. Impact depth for the momentum collimation}

For the collimation of beam halo, impact depth is a very important parameter. When the impact depth is very small, the collimation efficiency by one collimator is usually low due to the scattering effect from the inner surface of the collimator. In this case, the two-stage collimation method, in which a primary collimator scatters the halo particles and secondary collimators collect the scattered particles, is usually applied. However, the two-stage collimation method requires large phase advances between the primary and the secondary collimators and also between the secondary collimators. If the two kinds of collimators can be placed within one uninterrupted drift space, the primary collimator can be thick to absorb most of the hit particles, and this is considered the improved one-stage collimation method. The primary collimator and some secondary collimators are usually separated by magnets to obtain large phase advances, and it is better to use a thin primary collimator to produce a controlled scattering effect.

The impact depth for momentum collimation is a delicate parameter in an RCS. It varies between a few tens of micrometers and a few hundreds of micrometers. Here, a simple analysis is given to show the situation in the CSNS RCS. For the estimation of the radial precession per turn of a particle with a momentum deviation from the synchronous particle, we can make first-order estimation analytically.

The change in relative momentum spread per turn $(\Delta \delta)$ can be expressed by

$$
\Delta \delta=\frac{e V_{\mathrm{rf}}(\sin \phi-\sin \phi) s}{\beta^{2}\left(E+\varepsilon_{0}\right)},
$$

where $V_{\mathrm{rf}}, \varphi, \varphi_{s}, \beta, E$, and $\varepsilon_{0}$ are $\mathrm{rf}$ voltage, rf phase, synchronous phase, relativity velocity, kinetic energy, and rest energy, respectively. The particles to be collimated usually have the rf phase close to the synchronous phase as they are close to the bucket height (see Fig. 1) and have large momentum deviations. In the early acceleration phase in an RCS, the bucket height is usually about $1 \%$ in momentum spread depending on the rf voltage curve and the longitudinal injection scheme, which is about the same as defined by the momentum collimation system. For nontrapped particles, they may have larger momentum deviation than the bucket height and it may take many turns for them to hit the momentum collimators. However, only the particles resting in the very core of the horizontal emittance may have a momentum deviation as large as $-2 \%$ depending on the maximum normalized dispersion at the primary momentum collimator, and this will be explained in more

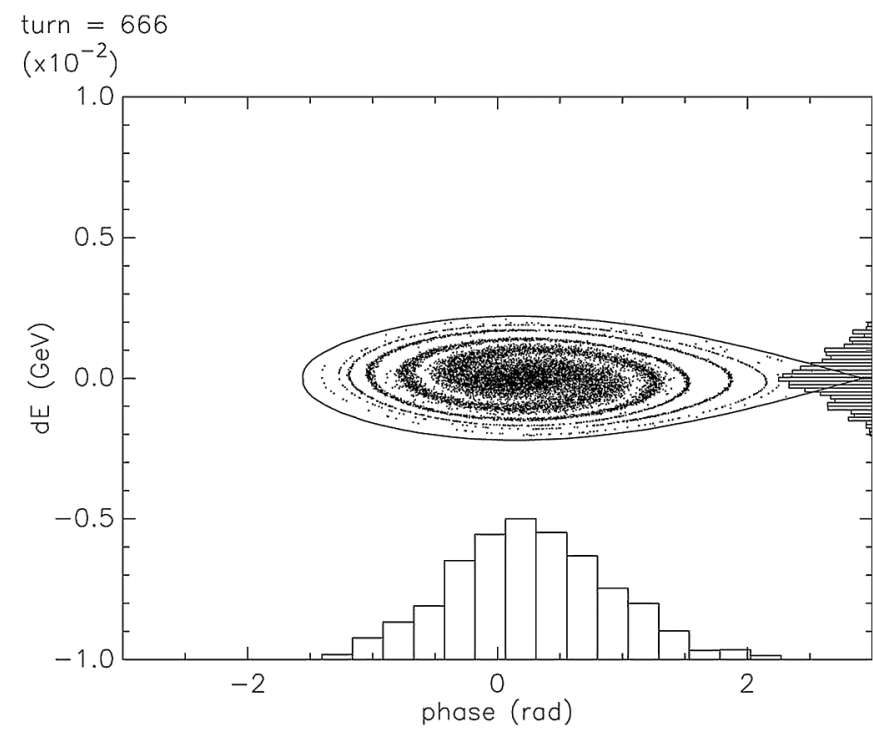

FIG. 1. One example for longitudinal beam distribution after rapid rf trapping (injection without chopping).

detail in Sec. IVA. There are two different scenarios for the momentum collimation: one is that the particle is still inside the rf bucket and the other is that the particle is outside the bucket (or nontrapped). In the former case, the precession in momentum deviation is relatively slow and the collimation always happens at a phase close to the synchronous phase. As an example (CSNS RCS, more details in Sec. IV), taking $V_{\mathrm{rf}}=100 \mathrm{kV}, \phi_{s}=10^{\circ}, E=$ $100 \mathrm{MeV}, D_{x}=3.2 \mathrm{~m}$, and $\phi=5^{\circ}$, thus from Eq. (1) we can estimate the precessions per turn in momentum deviation and in radial position as follows:

$$
\Delta \delta=4.3 \times 10^{-5} \text { and } \quad \Delta x=0.14 \mathrm{~mm} .
$$

For the particles with rf phases closer to the synchrotron phase, the precessions will become very small. Another factor of influencing $\Delta \delta$ is the growth rate of bucket height per turn, but it is usually negligible. A bunch height is smaller than the bucket height, but they are similar in the growth rate. However, the precession per turn in radial position is modulated by the betatron motion in the horizontal phase plane.

In the case of collimating nontrapped particles, the situation can be very different. The rf phase may be close to the synchronous phase or may not. Thus, the precession per turn in momentum deviation has a very large variation, with a maximum possible value of $\Delta \delta=5 \times 10^{-4}$, taking the above example. With a large precession per turn in momentum deviation, the radial precession per turn can reach the millimeter range and even the single-stage collimation method has very good collimation efficiency. It is often a similar situation for the survived particles from the transverse collimators, because they usually have large energy losses in the collimators. 

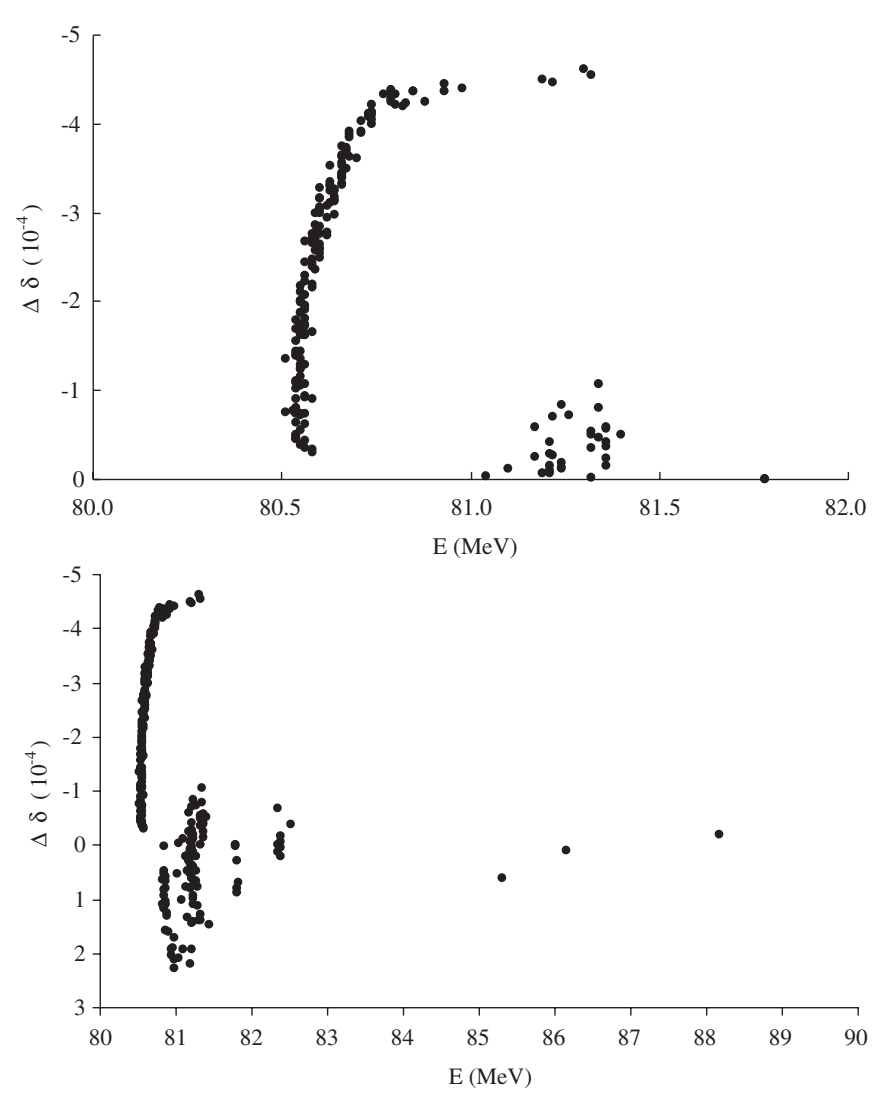

FIG. 2. Precession per turn in momentum deviation for the lost particles. Simulation conditions: CSNS RCS, 1D simulation, 0.83 chopping factor or $17 \%$ beam chopped, $0.1 \%$ offmomentum injection, a few particles of higher energy not shown. Upper: one-side collimation by $-1 \%$ in momentum deviation; lower: two-side collimation by $\pm 1 \%$ in momentum deviation.

Simulations with ORBIT code [5] can give the distribution of the momentum deviations for the lost particles. Figure 2 shows 1D simulation results with a single-side momentum collimation at $-1 \%$ and two-side collimation at $\pm 1 \%$, with a chopped injection (on-momentum, chopping factor: 0.83 ). From the simulation results, one can find, although most lost particles are nontrapped ones with larger precessions, there is still an important portion of the lost particles that are within the rf bucket and the precessions are smaller.

\section{B. Momentum collimation methods}

For large synchrotrons such as LHC at CERN and the main injector at FERMILAB, there are sufficient spaces to allocate a full two-stage momentum collimation system in one of the arcs or a dispersive region [8-10]. However, for compact synchrotrons, straight sections in arcs are much limited and there is no sufficient space to host a full twostage momentum collimation system. Thus, simplified momentum collimation methods are employed in this case. There are two reference methods for the momentum collimation in compact synchrotrons: the British spallation neutron source at Rutherford Appleton Laboratory (ISIS) method uses two-stage massive collimators and the J-PARC method uses a standard two-stage collimation. The former has two collimators within a long dispersive straight section, and produces a combined transversemomentum collimation effect. The latter places the primary momentum collimator at a location of high normalized dispersion in the arc section upstream of the transverse collimation section, and employs the whole transverse collimators as the secondary momentum collimators.

For the ISIS method [2], the study shows that with a phase advance of about $40^{\circ}$ in both transverse phase planes and a large dispersion it has reasonable collimation efficiency at relatively low energy such as $70 \mathrm{MeV}$ at ISIS. However, a long dispersive straight section is needed to obtain large phase advances. By coincidence, there are no dispersion-free straight sections at ISIS. In addition, the collimation efficiency will decrease dramatically with increasing energy, because the scattering effect on the inner surfaces of the first collimator becomes much more important at higher beam energy.

For the J-PARC method [3], it has not been put into operation at J-PARC. It has only thin tungsten foils as the primary momentum collimator at a high dispersive location. A preliminary study conducted here shows that it has significant drawbacks in collimating the particles with a large negative momentum deviation and a small horizontal Courant-Snyder invariant (or C-S invariant, hereafter as $I_{x}$ ). This is because, even with the scattering in the primary momentum collimator, the horizontal C-S invariant for these particles may still stay within the transverse collimation acceptance. In addition, some particles hitting the primary collimator will get lost in the downstream magnets before reaching the transverse collimators in the straight section due to Coulomb scattering, and become uncontrolled beam loss.

To overcome the drawbacks of the ISIS method and the J-PARC method, here we propose a new method called the combined momentum collimation method [11]. It has the following aspects: (i) combining two separate twostage collimation systems and a single-stage collimation system. A schematic of applying the method is given in Sec. IVA (Fig. 4). The primary momentum collimator is placed at a location of high normalized dispersion. The whole transverse collimation system in the dispersion-free straight section will be employed as the secondary collimators in one of the two-stage collimation systems, to collimate the scattered particles by the primary momentum collimators. The second two-stage collimation is composed of the primary momentum collimator and a secondary momentum collimator closely adjacent to the former, but the collection of the scattering particles is executed in successive turns. The single-stage collimation is that for some particles only the secondary momentum collimator at arc takes the effect. (ii) Using a thin foil (MP2 in Fig. 4) of higher atomic number (or high- $Z$ ) at negative $X$ (inner side 
of the reference orbit) as one part of the primary momentum collimator to produce significant scattering but with little energy loss, this can help minimizing the decrease in $I_{x}$ due to the longitudinal-horizontal coupling effect, so it facilitates the collection of the particles by the transverse collimators. (iii) A thin carbon foil is used (MP1 in Fig. 4) at positive $X$ to produce significant increase in $I_{x}$ while keeping a modest scattering. This will help the collection of the scattered particles by the transverse collimation system. (iv) A thick high- $Z$ jaw is used (MS1 in Fig. 4) as a secondary momentum collimator along with the thin high- $Z$ foil at the negative $X$ position of $0.5-1.0 \mathrm{~mm}$ further from the reference orbit. The betatron motion in successive turns can make the collimator effective in collecting the scattered particles having a small $I_{x}$ but a large momentum deviation. These particles are difficult to be collected by the transverse collimators. At the same time, this collimator also acts as a single-stage collimator to absorb the nontrapped particles with both a large momentum deviation and a large momentum turn precession, and also the particles survived from the transverse collimators but suffered a large energy loss there. (v) A second thick collimator (MS2 in Fig. 4) is located in the downstream arc section to localize some of the lost particles due to large angle scattering in the primary momentum collimator.

Another point to be addressed here is the dependence of momentum collimation efficiency upon beam correlation. The beam correlation comes from the injection painting in the phase spaces [12], usually either anticorrelated or correlated. For an anticorrelated beam, a particle with a large $I_{x}$ will have a small $I_{y}$; for a correlated beam, a particle with a large $I_{x}$ will have also a large $I_{y}$. Nevertheless the beam is correlated or anticorrelated, during the momentum collimation some particles with large $I_{y}$ have a probability to be lost in the downstream arc section before reaching the transverse collimators. However, the simulations in the next sections will show that with an anticorrelated beam the momentum collimation efficiency is significantly higher than with a correlated beam.

\section{BEAM-MATERIAL INTERACTION}

\section{A. Material choice for momentum collimators}

\section{Material choice for the primary momentum scraper}

Material choice for the primary momentum scraper is important here. We can find that different materials have different effects in energy loss and scattering angle. This means that, for different materials to obtain the same rms scattering angle, the relative changes in momentum due to the foil passing are different. For a high- $Z$ material, the relative change in momentum is smaller. For example, at the energy of $80 \mathrm{MeV}$, the average energy losses for the same rms scattering angle of $1.25 \mathrm{mrad}$ are 0.0148 and $0.1111 \mathrm{MeV}$ in tantalum and carbon, respectively. These correspond to the relative momentum change of
$1.0 \times 10^{-4}$ and $7.3 \times 10^{-4}$. This means that the relative momentum loss is only one-seventh in tantalum than in carbon. However, for different beam energies, if the rms scattering angle keeps the same, the relative change in momentum (or energy) almost does not change.

We can obtain the relation between the energy loss and the scattering angle for different materials from the theoretical formulas. The Bethe-Bloch formula for ionization loss is

$$
-\frac{d E}{d x}=\frac{4 \pi z^{2} e^{4}}{m_{e} \beta^{2} c^{2}} N Z B,
$$

where $N$ and $Z$ denote for the total number of atoms per unit volume in target and the atomic number of the target material, $B$ for the velocity-dependent correction factor, $z$ and $\beta$ for the charge and the Lorentz velocity of the projectile. For equivalent thickness $\xi$ (in $\mathrm{g} / \mathrm{cm}^{2}$ ), the ionization loss is

$$
-\frac{d E}{d \xi}=\frac{4 \pi z^{2} e^{4}}{m_{e} \beta^{2} c^{2}} N_{A} B \frac{Z}{A},
$$

where $N_{A}$ is the Avogadro constant and $A$ is the mass number of the target material.

The Moliere theory [13] for multiple scattering gives the rms scattering angle of a beam in a material:

$$
\theta_{m}=0.396 \frac{z Z}{p \beta c} \sqrt{\frac{\xi}{A} B^{\prime}},
$$

where $B^{\prime}$ is a correction factor.

The relation between the beam energy losses in different materials can be expressed

$\frac{\Delta E_{1}}{\Delta E_{2}}=\left(\frac{Z_{1}}{A_{1}} \xi_{1} / \sqrt{\frac{Z_{1}^{2} \xi_{1}}{A_{1}}}\right) /\left(\frac{Z_{2}}{A_{2}} \xi_{2} / \sqrt{\frac{Z_{2}^{2} \xi_{2}}{A_{2}}}\right)=\sqrt{\frac{A_{2}}{A_{1}} \frac{\xi_{1}}{\xi_{2}}}$.

To obtain the same scattering angle $\theta_{m}$ for different materials, we have

$$
\frac{\xi_{1}}{\xi_{2}}=\frac{A_{1} Z_{2}^{2}}{A_{2} Z_{1}^{2}}
$$

Thus,

$$
\frac{\Delta E_{1}}{\Delta E_{2}}=\frac{Z_{2}}{Z_{1}} .
$$

This relation can be easily confirmed by any Monte Carlo simulation code, such as TURTLE/MUSCAT [4]. From the $\beta$ dependence, one can find that the same relation holds for a wide range of energy as the parameter $B$ in Eq. (2) changes very slowly with $\beta$.

As mentioned in Refs. [14,15], the energy loss in a thin primary collimator at a dispersive location with a positive momentum deviation will increase $I_{x}$, which is helpful for the collection of the particle by transverse collimators. On the contrary, it will decrease $I_{x}$ if the momentum deviation 

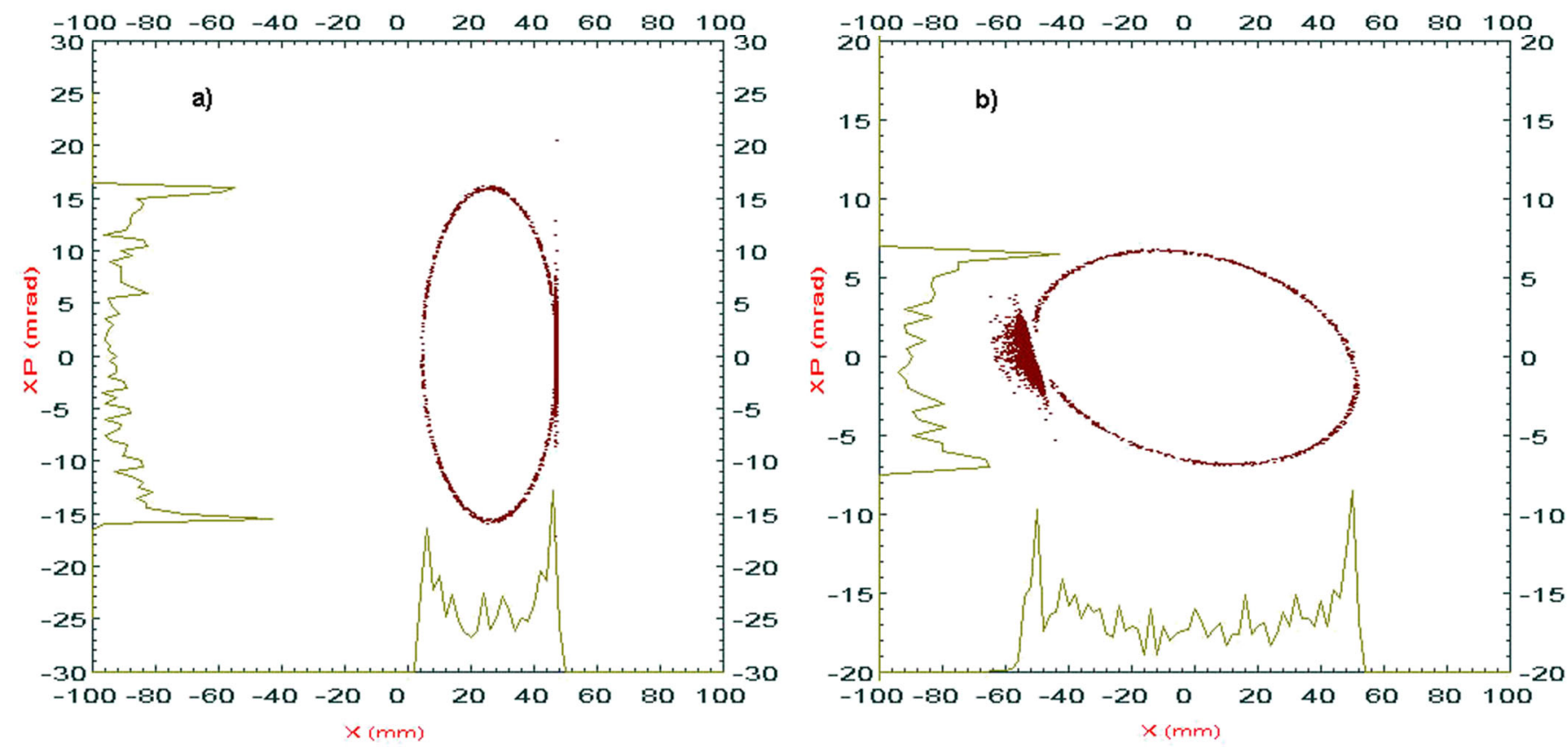

FIG. 3. Longitudinal-horizontal coupling effect in a primary momentum scraper (a) at the scraper; (b) at a dispersion-free location; CSNS RCS lattice, carbon foil of $0.05 \mathrm{~mm}$, at energy of $80 \mathrm{MeV}$, with a momentum deviation of $1.0 \%$.

is negative. It is the combined effect of scattering and longitudinal-horizontal coupling to determine if $I_{x}$ increases or decreases. In the following, the change in the $\mathrm{C}-\mathrm{S}$ invariant is derived.

Using normalized coordinates,

$$
\begin{aligned}
\xi & =\alpha_{x} x+\beta_{x} x^{\prime} \\
I_{x} & =\frac{1}{\beta_{x}}\left(x^{2}+\xi^{2}\right) \\
D_{x, n} & =D_{x} / \sqrt{\beta_{x}},
\end{aligned}
$$

where $D_{n}$ is normalized dispersion, assuming $D_{n}^{\prime}=0$ here. Suppose the change in $\Delta p / p_{0}$ (relative change in momentum) is small, then the change in the C-S invariant becomes

$$
\begin{aligned}
\Delta I_{x, p} & =\frac{1}{\beta_{x}}\left(2 x \Delta x+\Delta x^{2}+2 \xi \Delta \xi+\Delta \xi^{2}\right) \\
& \approx \frac{2}{\beta_{x}}(x \Delta x+\xi \Delta \xi) .
\end{aligned}
$$

In normalized coordinates, the scraper crossing is almost at $\xi \approx 0$, thus we have

$$
\Delta I_{x, p}=2 D_{x, n} \sqrt{I_{x}} \frac{\Delta p}{p_{0}}
$$

whereas the change in C-S invariant due to the scattering can be expressed by

$$
\begin{aligned}
\Delta I_{x, s} & =\frac{1}{\beta_{x}}\left(2 x \Delta x+\Delta x^{2}+2 \xi \Delta \xi+\Delta \xi^{2}\right) \approx \frac{1}{\beta_{x}} \Delta \xi^{2} \\
& =\beta_{x} \Delta x^{\prime 2}
\end{aligned}
$$

Thus, for the particles with large positive momentum deviations, a thin carbon foil at positive $X$ is a good choice to enhance the increase in $I_{x}$ due to relatively large energy loss with a modest scattering. While for the particles with large negative momentum deviations, a thin foil of tantalum or other high- $Z$ material at negative $X$ is a better choice to minimize the decrease in $I_{x}$ due to relatively small energy loss with the same scattering angle. The energy loss- $Z$ relation also means that a high- $Z$ material is better to reduce the heat deposit in a scraper. An example using TURTLE single-pass simulations is shown in Fig. 3, where a ring-type beam with a positive momentum deviation passed through a primary momentum collimator has an increased $I_{x}$ at a dispersion-free location, and scattering effect is also included. One can also see the increase in momentum spread induced by the scraper.

\section{Material choice for thick collimators}

If a thick block or collimator jaw will be used to absorb particles with relatively large impact depths, what material should we choose? This is the case of single-stage collimators or secondary collimators. In theory, to have a short stopping range and a small scattering angle per unit length, a material of large density and low- $Z$ is helpful. However, there is a roughly reverse tendency in the two properties in the periodic table of elements. The elements or their alloys in column VIII have heavier density than nearby elements. Table I shows the stopping ranges and lateral stragglings in carbon, nickel, copper, tantalum, and tungsten, calculated by using SRIM code [16]. By comparing the lateral straggling at the stopping end, one can find that nickel is the best 
TABLE I. Stopping ranges and lateral stragglings of different materials of proton beam at three different energies.

\begin{tabular}{lrrrrrrrrrrrrrrr}
\hline \hline & \multicolumn{1}{c}{$80 \mathrm{MeV}$} & \multicolumn{1}{c}{$130 \mathrm{MeV}$} & \multicolumn{4}{c}{$250 \mathrm{MeV}$} \\
\hline Material & $\mathrm{C}$ & $\mathrm{Ni}$ & $\mathrm{Cu}$ & $\mathrm{Ta}$ & $\mathrm{W}$ & \multicolumn{1}{c}{$\mathrm{C}$} & $\mathrm{Ni}$ & $\mathrm{Cu}$ & $\mathrm{Ta}$ & $\mathrm{W}$ & $\mathrm{C}$ & $\mathrm{Ni}$ & $\mathrm{Cu}$ & $\mathrm{Ta}$ & $\mathrm{W}$ \\
\hline Stopping range (mm) & 25.57 & 8.53 & 8.95 & 6.40 & 5.49 & 60.60 & 19.87 & 20.82 & 14.64 & 12.57 & 187.56 & 60.4 & 63.21 & 43.66 & 37.51 \\
Lateral straggling (mm) & 0.63 & 0.48 & 0.52 & 0.60 & 0.52 & 1.46 & 1.08 & 1.16 & 1.32 & 1.14 & 4.29 & 3.10 & 3.31 & 3.69 & 3.19 \\
\hline \hline
\end{tabular}

material for minimizing the surface scattering effect that particles escape from the inner surface before being fully stopped. The melting point of nickel as high as $1446^{\circ} \mathrm{C}$ also makes it a good candidate as the collimator material. The nickel-base alloy Inconel (e.g. Inconel 625: 62Ni$22 \mathrm{Cr}-9 \mathrm{Mo}-3.7 \mathrm{Nb}$ ) or tungsten or copper is also a good candidate. The collimation effect with these materials have been confirmed by simulations using ORBIT code. Certainly, the melting point and residual activation are also important in choosing the collimator material.

To have a good stopping efficiency in thick collimators, it is important to have a large ratio of the impact depth over the stopping range. For example, in the case of $80 \mathrm{MeV}$ in energy and $1 \mathrm{~mm}$ in impact depth, simulation shows that the collimation efficiency is almost $100 \%$ with a copper collimator. However, with a higher beam energy of $250 \mathrm{MeV}$, the collimation efficiency decreases to about $70 \%-75 \%$ for copper material due to the surface scattering. Therefore, tertiary or quarternary collimators are usually needed to absorb the surface scattered particles in highenergy synchrotrons. In case of limited available space for long absorbing collimators at higher beam energy, tungsten can be considered as it has a relatively short stopping range.

\section{B. Thickness choice for momentum collimators}

\section{Thickness of primary collimators}

The thickness of a primary collimator is chosen to obtain proper scattering angle or energy loss according to beam energy. The proper scattering can be determined by simulation studies. In this study with the CSNS input, it is found that, for the two-stage momentum collimation system with magnets crossed between the collimators, the scattering angle of about $1.25 \mathrm{mrad}$ in rms is a good choice, whereas the energy loss of about $0.1 \%$ for the positive momentum deviation can be a good benefit and the energy loss of less than $0.02 \%$ for the negative momentum deviation can be considered an acceptable drawback. Table II shows the thicknesses of the primary momentum collimators at different energies.

\section{Thickness of thick collimators}

A secondary collimator is supposed to absorb all the particles hitting on it, and its thickness or length is decided by the stopping range in the collimator material that increases with the beam energy. As discussed in Sec. III A 2, nickel or tungsten is a good material for secondary collimators. Considering the collimation is to cover a range of beam energy, the thickness of the secondary collimator is taken somewhat larger than the calculated thickness for the nominal maximum energy for collimation. Tungsten is applied as the material for the secondary collimators in the simulations here, as nickel is not available in the ORBIT code. Therefore the real collimation efficiency may be slightly better when nickel is used in the machine.

\section{Technical issues concerning the momentum collimators}

In all high-intensity hadron synchrotrons, the technical issues including cooling, induced radioactivity, radiation shielding, and mechanical driving should be considered in the design of collimators. These should be considered together with the physical performance of the collimators. Here the cooling property and the induced radioactivity for the momentum collimators are discussed due to its connection with the material selection. The total heat deposit in a collimator is related to the collimation design and the total beam power collected by the collimators. A total collimated beam power of a few kilowatts is usually designed in high-power proton synchrotrons to allow the collimation efficiency of $90 \%-95 \%$ and the uncontrolled loss rate of $1 \mathrm{~W} / \mathrm{m}$ for hands-on maintenance. The majority of the collimated beam power is deposited in secondary collimators, but the cooling in secondary collimators is

TABLE II. Thickness of the primary momentum collimators at different energies.

\begin{tabular}{llllllc}
\hline \hline Beam energy $(\mathrm{MeV})$ & \multicolumn{2}{c}{80} & \multicolumn{2}{c}{130} & \multicolumn{2}{c}{250} \\
\hline Material & \multicolumn{1}{c}{ Ta } & \multicolumn{1}{c}{$\mathrm{C}$} & $\mathrm{Ta}$ & $\mathrm{C}$ & $\mathrm{Ta}$ & $\mathrm{C}$ \\
\hline Thickness $(\mu \mathrm{m})$ & 1.3 & 50 & 2.7 & 120 & 7.4 & 370 \\
rms scattering angle (mrad) & 1.253 & 1.246 & 1.251 & 1.254 & 1.256 & 1.246 \\
Energy loss $(\mathrm{MeV})$ & 0.0096 & 0.0694 & 0.0143 & 0.1164 & 0.0260 & 0.2332 \\
\hline \hline
\end{tabular}



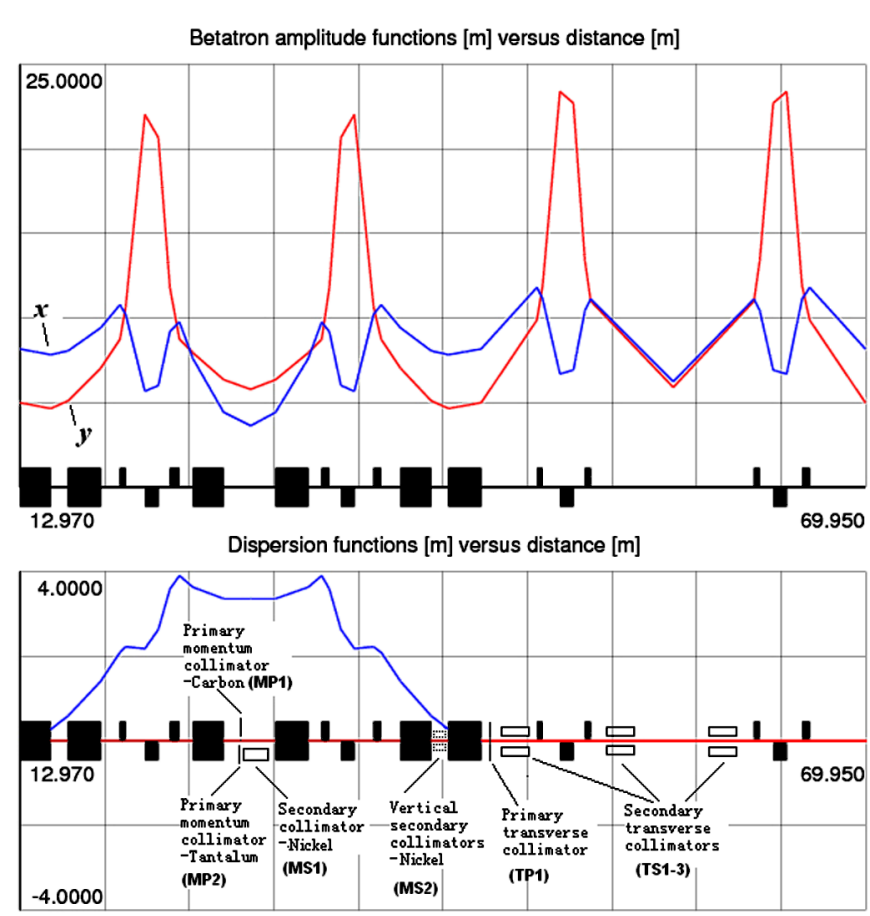

FIG. 4. Lattice structure and the layout of the transverse collimators and the momentum collimators at the CSNS RCS.

usually less problematic due to their large sizes and relatively dispersed heat deposition so that it is easy to design a water- or a fans-cooling system. For some secondary or tertiary collimators with low heat deposit, natural cooling is just sufficient. On the contrary, although the heat deposit in primary collimators is not large, their small thickness and very concentrated and pulsed heat deposit to the blades may introduce problems to the cooling design of the collimators. Usually indirect water cooling or fans cooling is used for primary collimators. It has to be ensured that the hottest point should be far below the melting temperature of the material.

Carbon is a good material in heat resistance and has less induced radioactivity; thus, it is an ideal material as the primary momentum collimator MP1 as shown in Fig. 4. As for the primary momentum collimator MP2, tantalum is also a good heat-resistant material but its heat conductivity is poor; therefore in some cases tungsten may be used for its better heat conductivity. The melting point and thermal conductivity for nickel are $1728 \mathrm{~K}$ and about $90 \mathrm{~W} / \mathrm{m} / \mathrm{K}$, and they are acceptable as secondary collimators. Because usually the collimators are locally shielded, the induced radioactivity is not very important here.

\section{SIMULATIONS OF THE COMBINED MOMENTUM COLLIMATION METHOD}

\section{A. Momentum collimation scheme at the CSNS RCS}

The CSNS RCS has been chosen for the studies of the combined momentum collimation method. It has a lattice of all-triplet cells, and its main parameters are shown in Table VIII in the Appendix. The lattice is very suitable for making both transverse and momentum collimations. Based on the combined momentum collimation method discussed above, a momentum collimation scheme for the RCS has been studied. Figure 4 shows the layout of the collimators. Table III gives some key information for the collimation system.

The nominal acceptance for the primary transverse collimators is $350 \pi \mathrm{mm} \mathrm{mrad}$, and the one for the primary momentum collimators is $350 \pi \mathrm{mm}$ mrad plus $\pm 1 \%$ in momentum deviation, whereas the nominal physical acceptance of the ring is $540 \pi \mathrm{mm}$ mrad plus $\pm 1 \%$ in momentum deviation. However, as mentioned above, some nontrapped particles with a much smaller $I_{x}$ but a larger momentum deviation will also hit the primary momentum collimator. With the normalized dispersion of $1.78 \mathrm{~m}^{1 / 2}$ $\left(D_{x}=3.3905 \mathrm{~m}, \beta_{x}=3.9503 \mathrm{~m}\right)$ at the primary momentum collimator, the maximum momentum deviation still clearing from the collimators is $\pm 2.1 \%$. As we do not apply an rf bucket higher than $1.2 \%$ in momentum spread during the acceleration, the theoretical momentum ranges for momentum collimation are from $-2.1 \%$ to $-1.0 \%$ and from $1.0 \%$ to $1.2 \%$. As mentioned above, the particles with a large negative momentum deviation and a small $I_{x}$ will be collimated mainly by the tantalum scraper and the nickel or tungsten absorber (MS1) in the successive turns. A small part of them with a large momentum precession will be collimated directly by MS1 as a one-stage collimator; and the collimation efficiency is expected to be not too low due to a relatively large impact depth in this case. However, the efficiency decreases with increasing beam energy due to the surface scattering effect as discussed in Sec. III A.

TABLE III. Collimators used for the combined momentum collimation method studies. (Note: The phase advances for MS1 are for the next turn with given tunes of 5.82/5.80).

\begin{tabular}{lccll}
\hline \hline & Material & $\beta_{x} / \beta_{y}(\mathrm{~m})$ & $D_{x}(\mathrm{~m})$ & $\Delta \mu_{x} / \Delta \mu_{y}\left({ }^{\circ}\right)$ \\
\hline Primary momentum collimator $(\delta>0)-M P 1$ & Carbon & $3.950 / 5.608$ & 3.391 & $0.0 / 0.0$ \\
Primary momentum collimator $(\delta<0)-M P 2$ & Tantalum & $3.950 / 5.608$ & 3.391 & $0.0 / 0.0$ \\
Secondary momentum collimator MS1 & Nickel & $3.950 / 5.608$ & 3.391 & $295.2 / 288.0$ \\
Secondary momentum collimator MS2 & Nickel & $7.488 / 5.223$ & 0.542 & $108.5 / 88.8$ \\
Primary transverse collimator TP1 & Blackbody & $12.292 / 9.257$ & 0. & $153.2 / 157.7$ \\
\hline \hline
\end{tabular}




\section{B. Simulations of the combined momentum collimation method with hollow beams}

\section{Simulation conditions and beam correlation}

As the scattering process introduces a correlation between the horizontal and the vertical phase planes, and the longitudinal-horizontal coupling if it happens at a dispersive location, hollow or ring-type beams in the phase spaces have been used in the simulations to identify the collimation effectiveness of different particles by different collimation mechanisms. Other reasons to employ hollow beams here are as follows: the beam in a real RCS is usually either correlated or anticorrelated due to the injection painting in the phase spaces; one method to obtain good statistical results with relatively less macroparticles is to track only the potentially lost particles as possible. A self-made FORTRAN program (HOLLOW BEAM) was developed to generate hollow beam distributions with different correlations.

Both TURTLE and ORBIT codes have been used to study the details, with the first showing the one-pass collimation effect and the second showing also the dynamic collimation effect including acceleration and multiturn effect. The initial hollow beam distributions are input from an outside file generated by HOLLOW BEAM.

Assuming the particles to be collimated by the momentum collimation system first hit the primary momentum scrapers, and then they are absorbed by the secondary collimators in the successive turns. To simplify the calculations here, it is supposed that all the particles hitting on
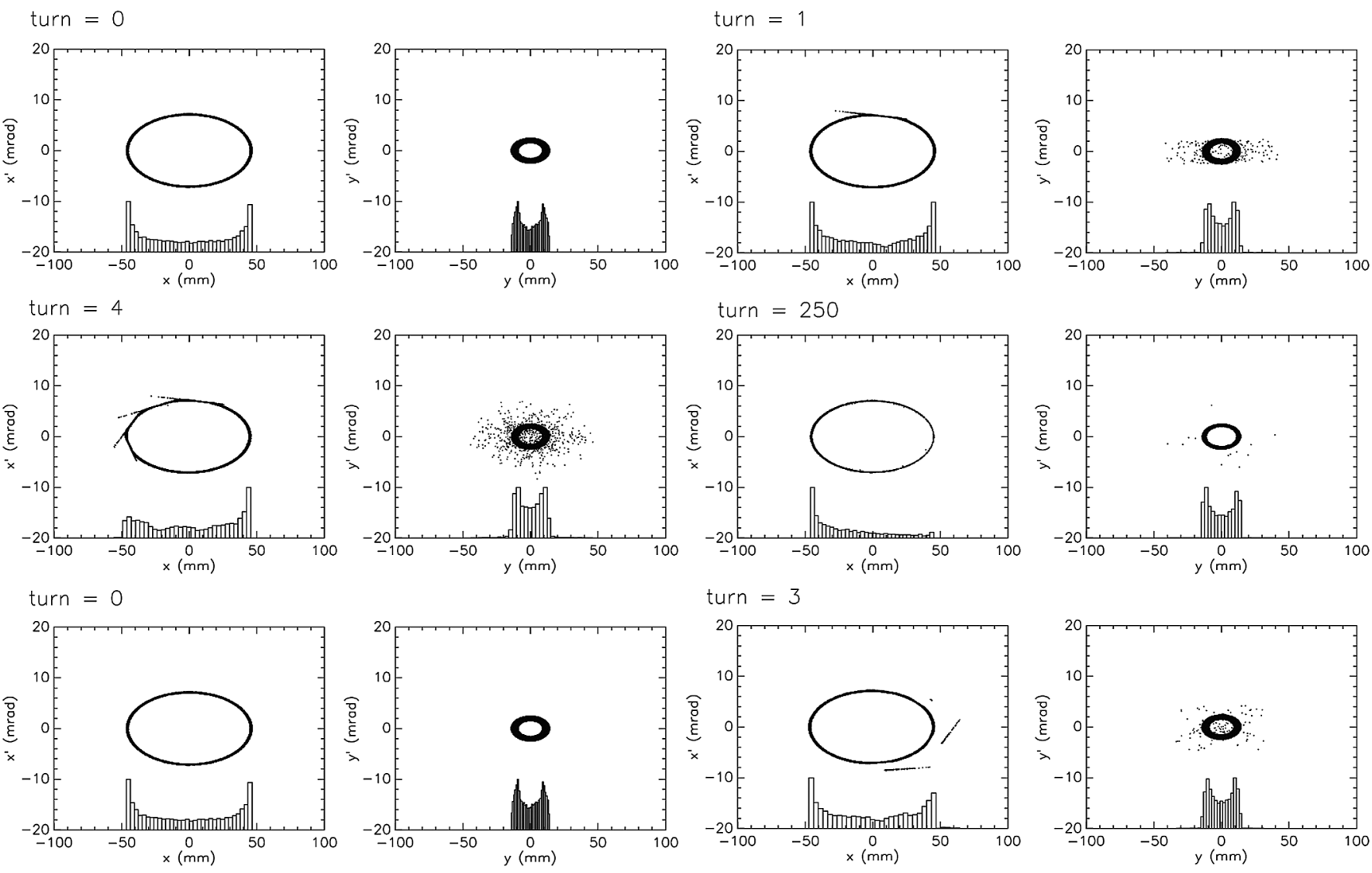

turn $=3$
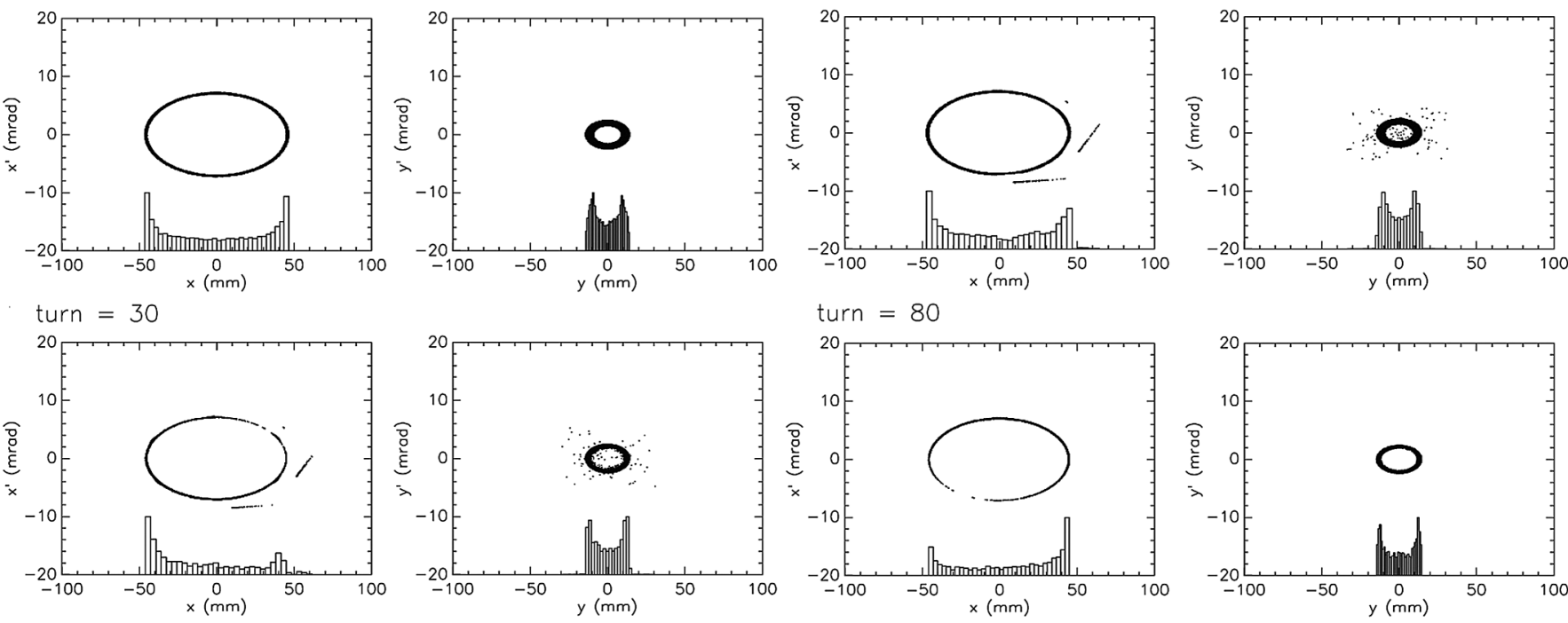

\section{turn $=80$}
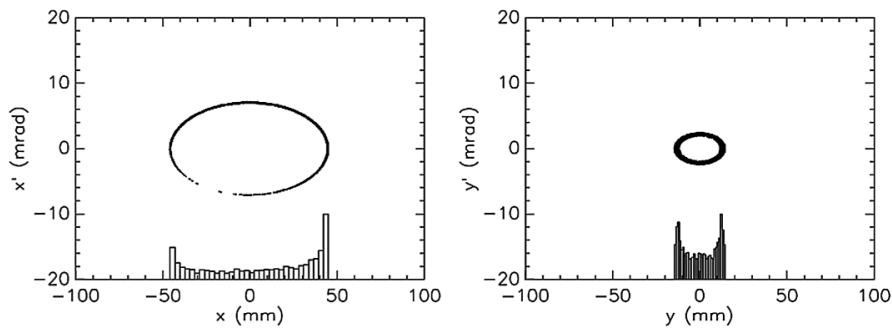

FIG. 5. Beam distributions at the middle point of the dispersion-free long straight section at different turns during the halo collimation of an anticorrelated beam at $80 \mathrm{MeV}$. Each pair of plots with one in the horizontal plane and the other in the vertical planes corresponds to the given turn. Upper part (four pairs): $\delta=-1.03 \%$; lower part (four pairs): $\delta=1.03 \%$. 

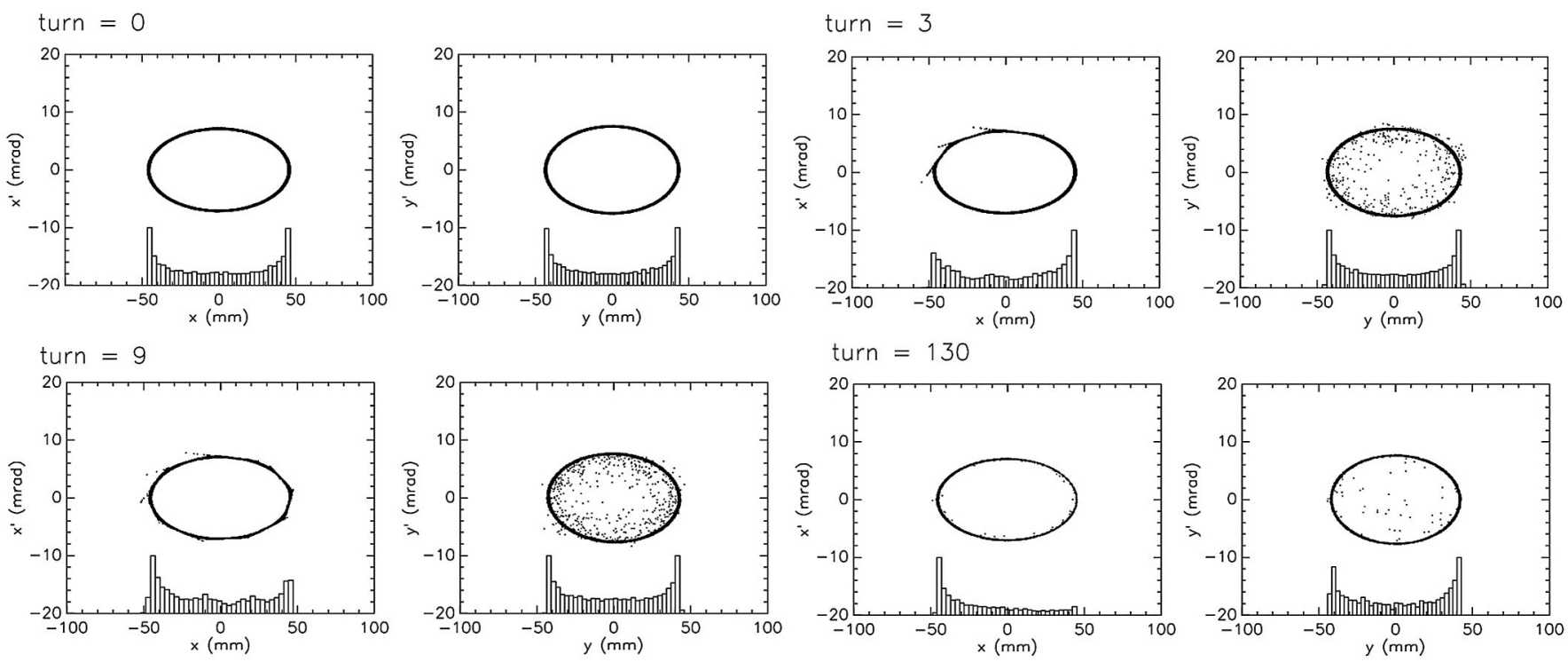

FIG. 6. Beam distributions at the middle point of the dispersion-free long straight section at different turns during the halo collimation of a correlated beam at $80 \mathrm{MeV}(\delta=-1.03 \%)$.

the transverse collimation system will be collimated with a fixed efficiency of $100 \%$ or $95 \%$. As the collimation mechanisms for the halo and core particles in the horizontal phase plane are different, the simulations for the two cases are carried out separately. For example, the emittance ring for the beam halo is taken as from $90 \%$ to $96 \%$ of the collimation acceptance $(350 \pi \mathrm{mm} \mathrm{mrad})$, and the one for the beam core is taken as between $0 \%$ to $6 \%$ of the collimation acceptance. The thickness of the emittance ring of $6 \%$ for the beam halo corresponds to about $1.0 \mathrm{~mm}$ in dimension at the scraper.
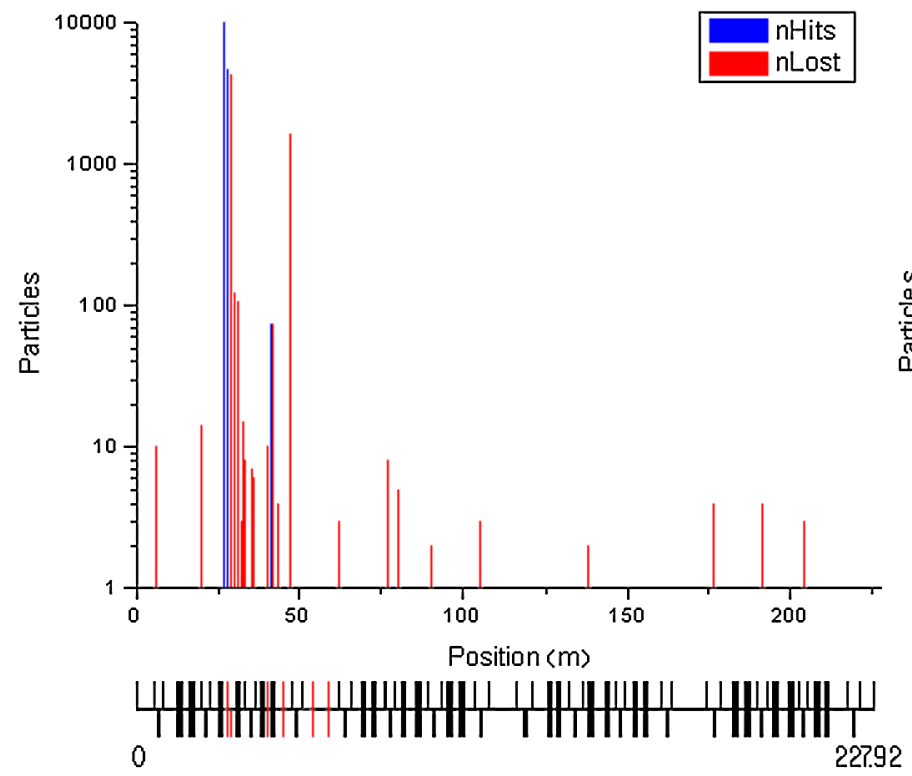

As pointed out in Sec. II B, the beam correlation coming from the injection painting has an important impact on collimation efficiency. Simulations for both anticorrelated and correlated beams have been carried out. Although in reality the beam correlation becomes more complicated when the space charge effect is taken into account, this study does not lose its generality.

In order to identify the collimation mechanisms and their collimation efficiencies for different particles, just coasting beams have been used in the simulations in this section.

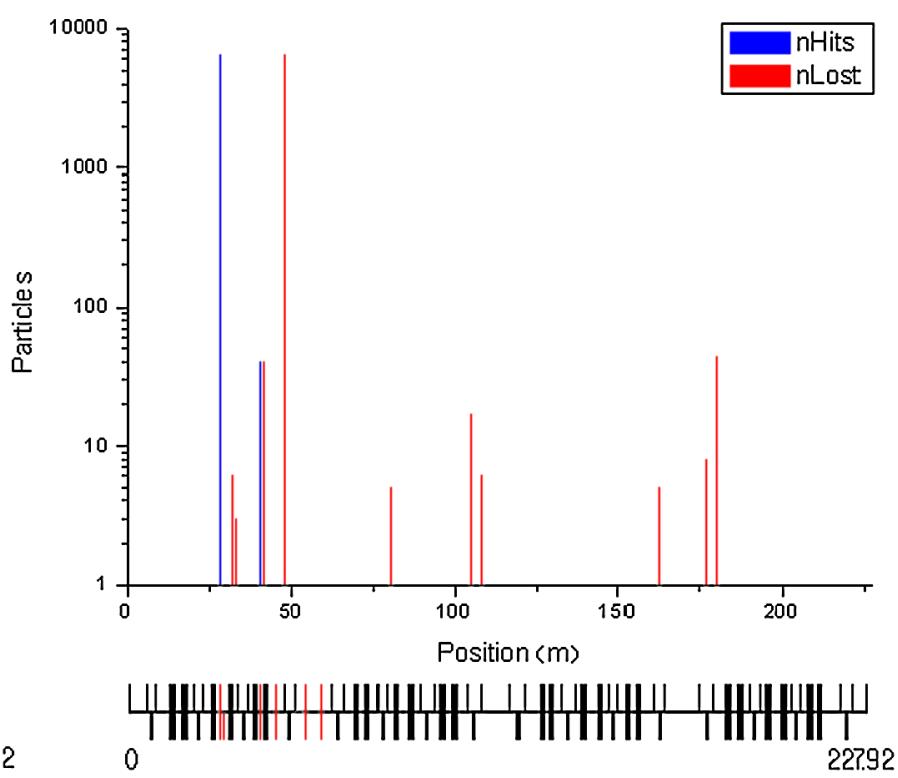

FIG. 7. Lost particles and the corresponding positions in the ring for an anticorrelated beam at $80 \mathrm{MeV}$. nHits denotes the number of particle hitting on the collimators and nLost denotes the number of absorbed particles. A total of 10000 particles are injected. Left: $\delta=-1.03 \%$; right: $\delta=1.03 \%$. 

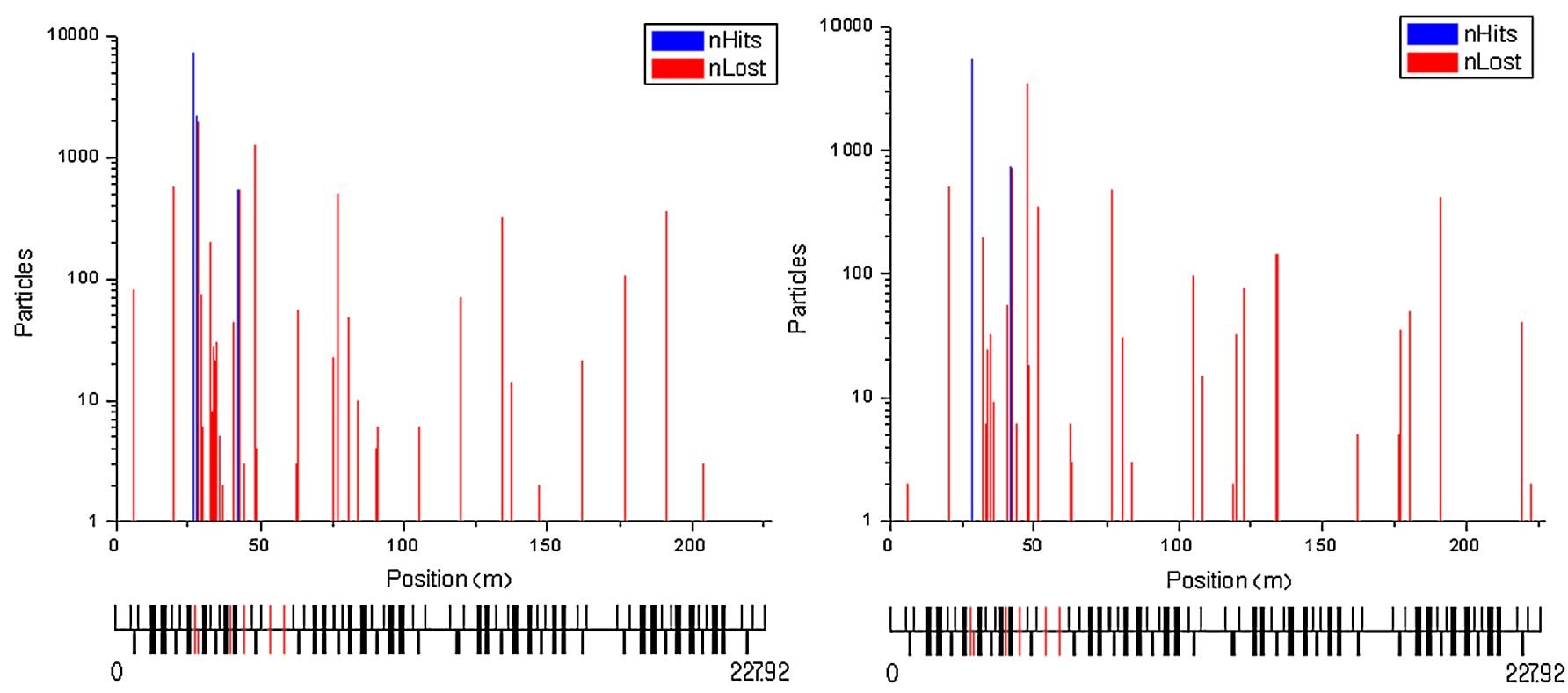

FIG. 8. Lost particles and the corresponding positions in the ring for a correlated beam at $80 \mathrm{MeV}$. nHits and nLost have the same meaning as in Fig. 4. A total of 10000 particles are injected. Left: $\delta=-1.03 \%$; right: $\delta=1.03 \%$.

\section{Simulation results with coasting beams}

First, the simulation results on the momentum collimation of the horizontal beam halo are presented. Figures 5 and 6 show the distributions during the collimation process for both anticorrelated and correlated beams. For an anticorrelated beam, the collimation process is faster for the particles with positive momentum deviations than for the particles with negative momentum deviations, and the reason is that the increase in $I_{x}$ due to the energy loss in the momentum scraper is more significant than by the scattering effect. Faster collimation means less turns needed. For a correlated beam, there are some particles remaining within the ellipse after the collimation in the case of negative momentum deviation because the combined effect of the scattering and the energy loss in the primary momentum collimator will result in a decrease in $I_{x}$ for some particles. Figures 7 and 8 show the numbers of the lost and hitting particles at the collimators or other ring devices with respect to their positions in the ring. Then the same results for the horizontal beam core are shown in Figs. 9 and 10. Some results are also summarized in Table IV.

From the simulation results, it can be seen that the collimation efficiency with an anticorrelated beam is much higher than that with a correlated beam, except for
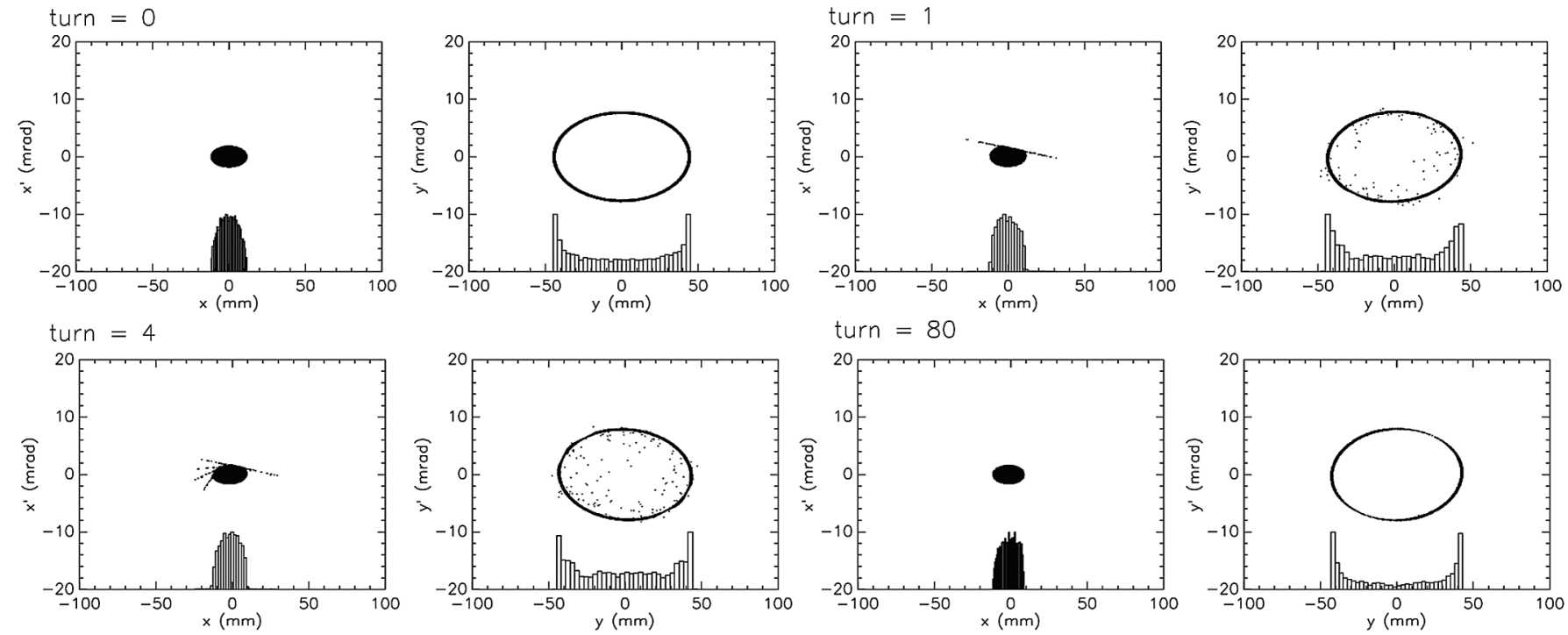

FIG. 9. Beam distributions at the middle point of the dispersion-free long straight section at different turns during the core collimation ( $\delta=-1.85 \%$, anticorrelated beam, $80 \mathrm{MeV})$. 

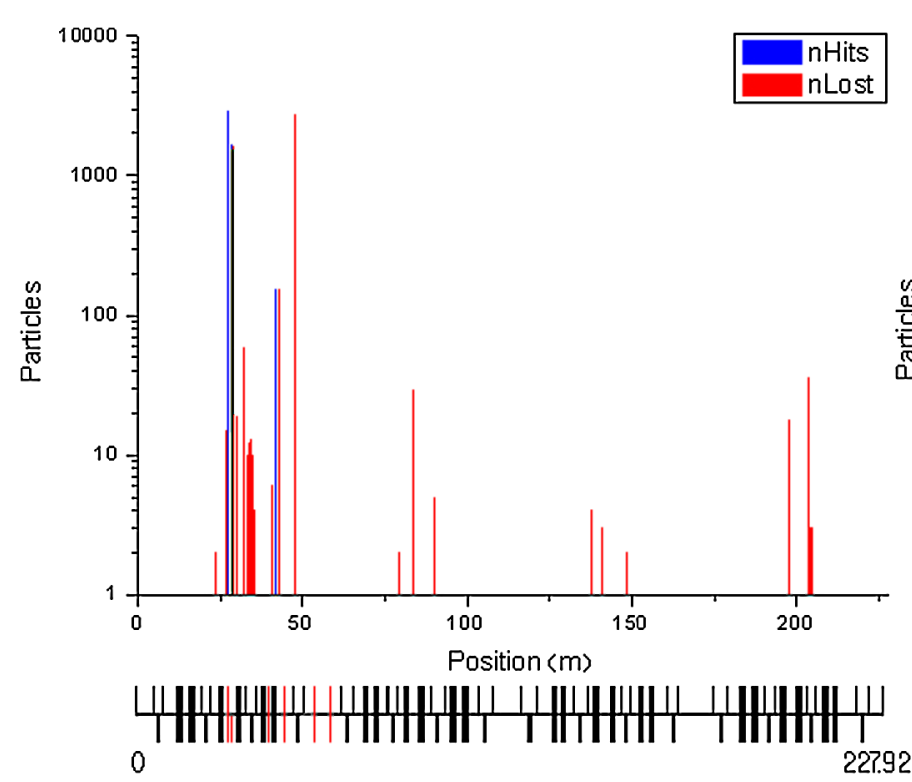

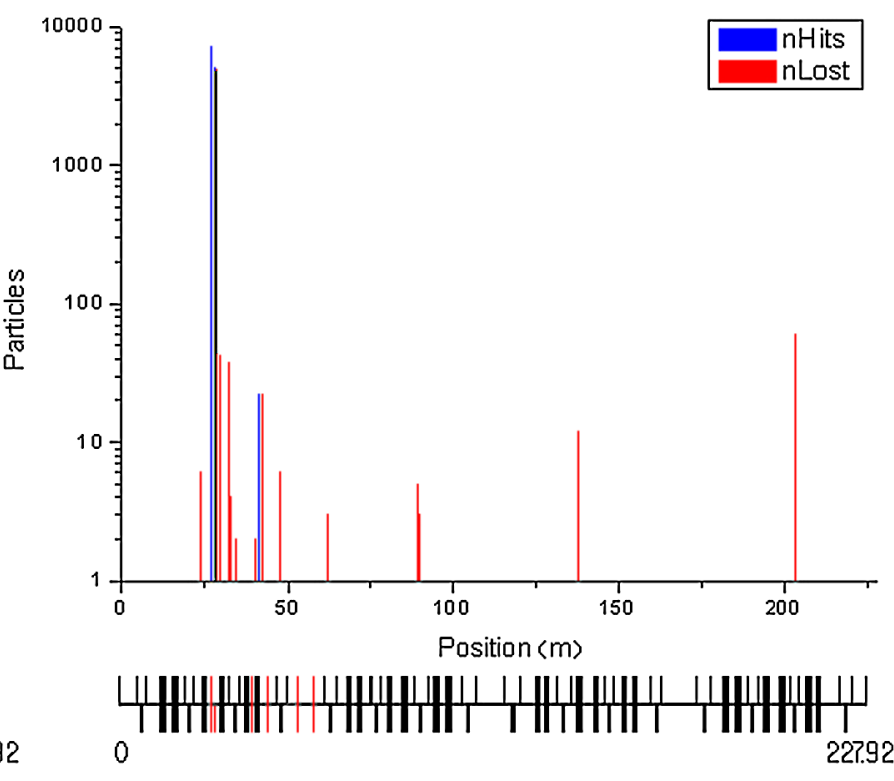

FIG. 10. Lost particles and the corresponding positions in the ring for the core collimation $(\delta=-1.85 \%)$ at $80 \mathrm{MeV}$. nHits and nLost have the same meanings as in Fig. 4. A total of 10000 particles are injected. Left: anticorrelated beam; right: correlated beam.

the innermost particles with very small $I_{x}$. The main reason is that the scattering in the primary momentum collimator increases the C-S invariants in both the transversal phase planes, but with a correlated beam the outer particles with large initial $I_{y}$ will have a large probability to be lost in the downstream arc section before reaching the transverse collimators. Because the beam profile is close to being rectangular in high dispersive regions, some particles are lost in the ceramic vacuum chambers that are either race-track type (for dipole magnets) or circular type (for quadrupoles).

It is also evident that for the particles with positive momentum deviations the collimation mechanism has a very good efficiency. This is assured by the significant increase in $I_{x}$ for all the particles passing through the carbon foil and can be easily collimated by the transverse collimators. As the beam core with the maximum positive momentum deviation of $1.2 \%$ will stay clear from the primary momentum collimator, only the beam halo part needs to be considered here.

For the particles with negative momentum deviations, the collimation mechanisms are different for the beam core and the beam halo in the horizontal phase plane. For the former, the secondary collimation is mainly performed by the thick nickel or tungsten block (CP1) and it has a very high efficiency. For the latter, the secondary collimation is by the combined effect of the transverse collimators and $\mathrm{CP} 1$. It is interesting to see that the collimation efficiency for the beam core of a correlated beam is very high. In this case, one can ignore the scattering in the vertical phase plane when studying the collimation process.

Therefore, one can say that with an anticorrelated beam the combined momentum collimation method has very good collimation efficiency for both the beam core and the beam halo.

\section{Further analysis on the collimation efficiencies of beam halo and beam core}

From Table III, one can find that the collimation efficiency of the beam core is higher than the beam halo. The reason is depicted in Fig. 11. When hitting the momentum scraper, a particle in the beam core has a larger momentum deviation than a particle in the beam halo. Because of the dispersion, the scattered particles of the beam core will

TABLE IV. Collimation efficiencies and turns to collimate all the particles hitting the primary momentum collimator with the combined collimation method at the energy of $80 \mathrm{MeV}$, with the transverse collimation efficiency taken as $100 \%$.

\begin{tabular}{|c|c|c|c|c|}
\hline \multirow[b]{2}{*}{$\mathrm{C}-\mathrm{S}$ invariant $(\pi \mathrm{mm} \mathrm{mrad}) /$ off- momentum } & \multicolumn{2}{|c|}{ Anticorrelated } & \multicolumn{2}{|l|}{ Correlated } \\
\hline & Collimation efficiency & Turns & Collimation efficiency & Turns \\
\hline$I_{x}=333, \delta=1.03 \%$ & $98.4 \%$ & 80 & $61.9 \%$ & 70 \\
\hline$I_{x}=333, \delta=-1.03 \%$ & $94.1 \%$ & 250 & $58.8 \%$ & 130 \\
\hline$I_{x}=18, \delta=-1.85 \%$ & $94.3 \%$ & 80 & $96.2 \%$ & 100 \\
\hline
\end{tabular}




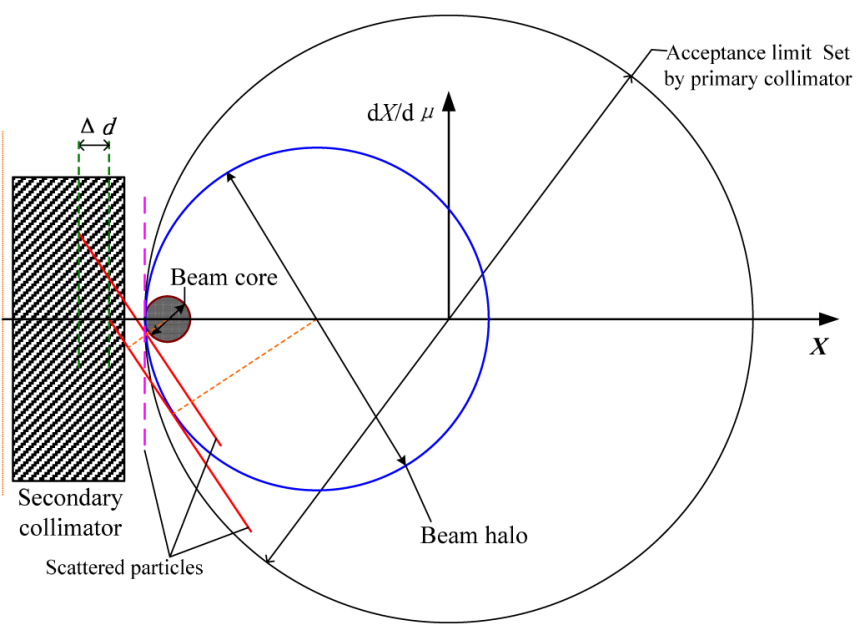

FIG. 11. Impact depth of scattered particles at the secondary momentum collimator is higher for beam core than for beam halo (in normalized emittance).

appear a little farther out at the secondary momentum collimator in successive turns than the ones of the beam halo. Therefore, the impact depth in the case of the beam core is larger than in the case of the beam halo, and the difference is $\Delta d$ in Fig. 11.

\section{APPLICATION OF THE COMBINED MOMENTUM COLLIMATION METHOD TO THE CSNS RCS}

\section{A. Collimation system at the CSNS RCS}

The collimation system at the CSNS RCS is shown in Fig. 4. A dedicated dispersion-free long straight section of one longer drift of $11 \mathrm{~m}$ and two shorter drifts of $3.8 \mathrm{~m}$ is reserved for the transverse collimation. Actually only $11 \mathrm{~m}$ long drift is used in the design [17], but it may be changed to use two long drifts as shown in Fig. 4 in the future. As discussed in the previous sections, the transverse collimators also play roles in the momentum collimation. To simplify the study, the transverse collimators are presented by a single blackbody absorber with a fixed collimation efficiency of either $100 \%$ or $95 \%$. In principle, due to the prescattering in the primary momentum collimators, the
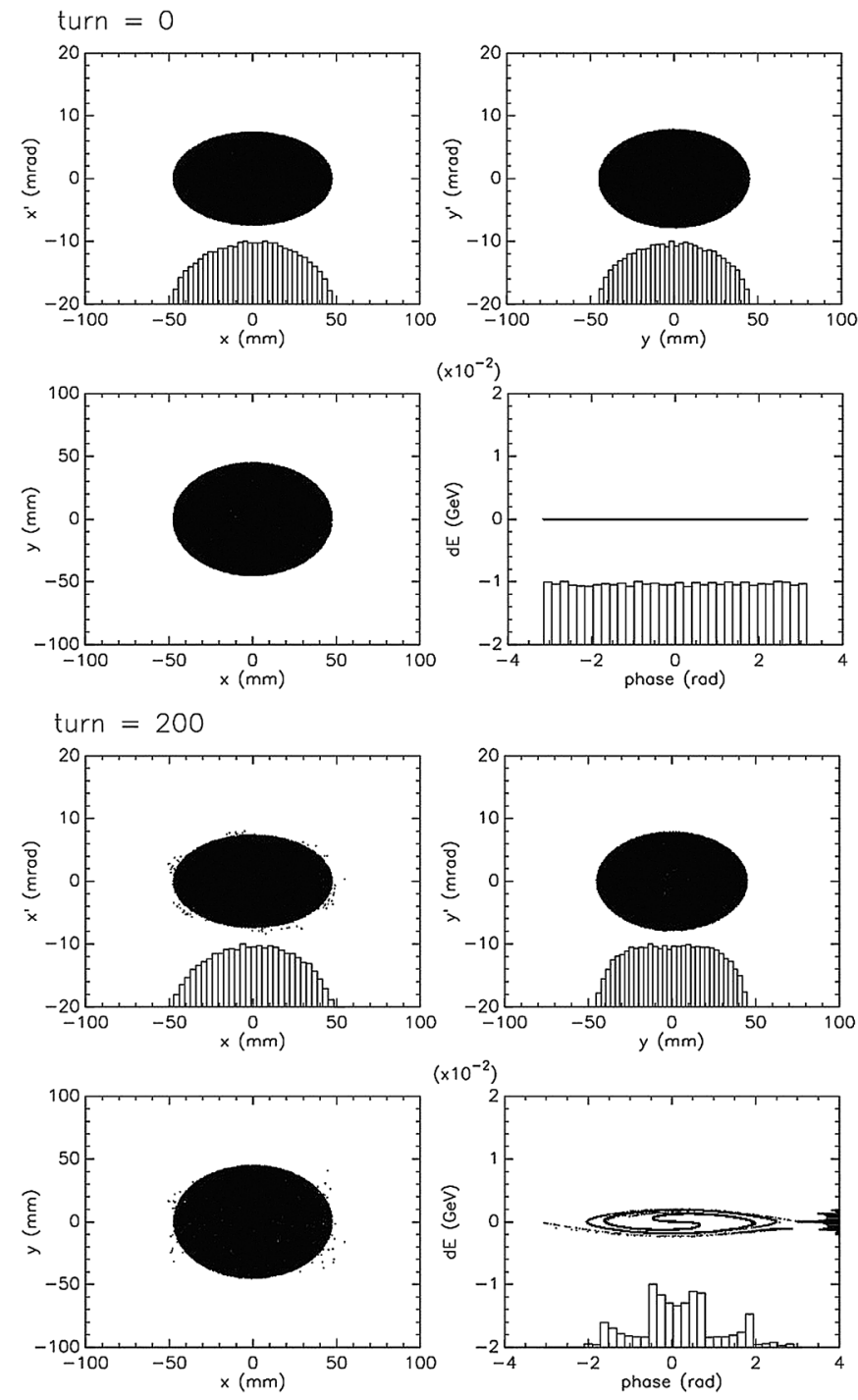

FIG. 12. Distributions in the phase spaces with one-turn injection and after some turns at the injection point with an anticorrelated beam. The injection energy is $80 \mathrm{MeV}$. Upper: at injection; lower: after 200 turns.

whole transverse collimators as the secondary collimation should have a larger efficiency than the transverse collimation itself where the collimation efficiency is expected to be about $95 \%$.

TABLE V. Parameters of the momentum collimators for the CSNS RCS.

\begin{tabular}{|c|c|c|c|c|}
\hline \multirow{2}{*}{$\frac{\text { Collimators }}{\text { Material }}$} & \multicolumn{2}{|c|}{ Primary } & \multicolumn{2}{|c|}{ Secondary } \\
\hline & Ta foil & C foil & 1st W block & 2nd W block \\
\hline Longitudinal position (m) & 28.465 & 28.465 & 28.515 & 40.910 \\
\hline \multirow[t]{3}{*}{ Thickness (mm) } & 0.0013 & 0.05 & 30 & 30 \\
\hline & 0.0027 & 0.12 & 50 & 50 \\
\hline & 0.0074 & 0.37 & 100 & 100 \\
\hline Shape & Erect & Erect & Erect & Erect \\
\hline Transverse position & $-X$ & $+X$ & $\pm X$ & $\pm Y$ \\
\hline Off $X$ axis (mm) & -71.08 & +71.08 & \pm 71.08 & \pm 45.79 \\
\hline
\end{tabular}


TABLE VI. Overall collimation efficiencies of the momentum collimation system at the CSNS RCS.

\begin{tabular}{|c|c|c|c|c|c|c|}
\hline \multirow{2}{*}{$\begin{array}{l}\text { Correlation } \\
\text { Energy }(\mathrm{MeV})\end{array}$} & \multicolumn{3}{|c|}{ Anticorrelated } & \multicolumn{3}{|c|}{ Correlated } \\
\hline & $80-90$ & $130-140$ & $250-260$ & $80-90$ & $130-140$ & $250-260$ \\
\hline Efficiency $\left(\%, \eta_{\mathrm{tr}}=100 \%\right)$ & 95.4 & 96.8 & 92.5 & 63.9 & 49.4 & 36.0 \\
\hline Efficiency $\left(\%, \eta_{\mathrm{tr}}=95 \%\right)$ & 92.6 & 93.9 & 89.3 & 62.4 & 48.2 & 34.9 \\
\hline Efficiency $\left(\%, \eta_{\mathrm{tr}}=95 \%\right.$, with a tertiary collimator $)$ & $\cdots$ & $\cdots$ & 91.8 & $\cdots$ & $\cdots$ & 35.0 \\
\hline
\end{tabular}

To show the effectiveness of the combined momentum collimation method, different beam energies that correspond to different CSNS construction phases have been used in the studies. With the increasing beam energy, all the momentum collimators especially the primary scrapers should be replaced by thicker ones to maintain the similar effect. However, the surface scattering effect in secondary collimators becomes more important at higher beam energy. Table $\mathrm{V}$ gives the parameters of the momentum collimators at three CSNS phases. Similar to the studies presented in Sec. IV, tungsten instead of nickel is used as the material for the secondary collimators in the simulations.

\section{B. Simulation results of the momentum collimation system}

Not like the simulations presented in Sec. IV where hollow and coasting beams are used, here more realistic beam conditions including the rf trapping and acceleration are used to study the overall collimation effect.

In the simulation studies with ORBIT, input beams with both anticorrelated and correlated distributions in the transverse planes are also generated by the HOLLOW BEAM code. Elliptic shape and uniform distributions in both the trans-
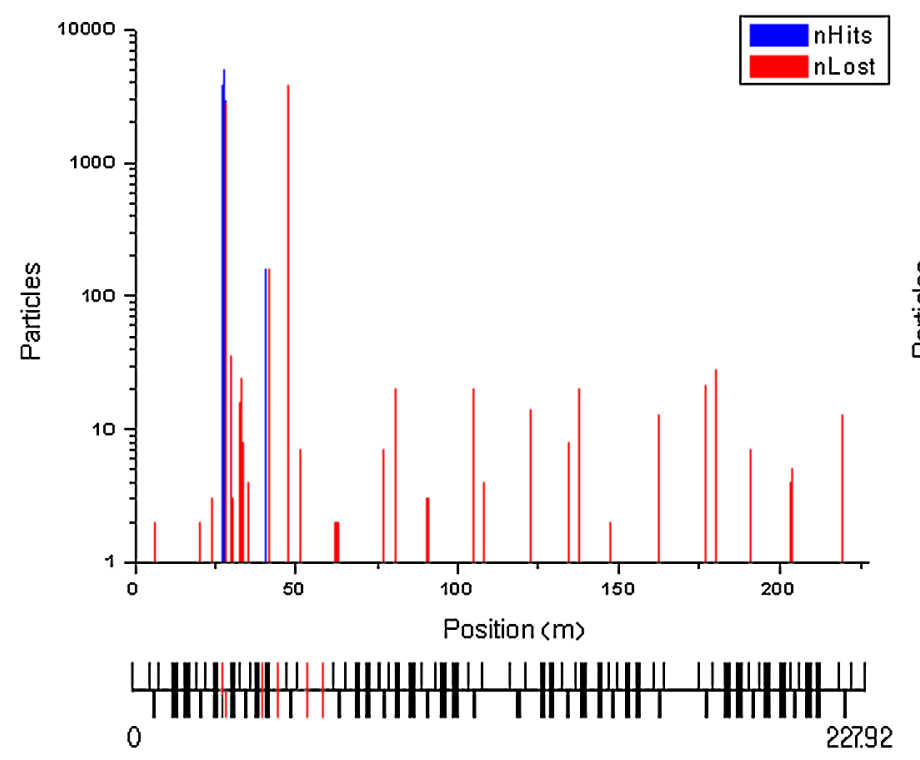

verse phase spaces and zero-emittance uniform distribution in the longitudinal phase space are used. The maximum transverse emittance is chosen as $350 \pi \mathrm{mm}$ mrad. Because these studies are to show the collimation efficiency of the proposed method, the total beam loss rate is not important here. To obtain a sufficiently statistical effect by having more particles involved in the momentum collimation, together with the nonchopping injection the rf voltage pattern is also designed to produce relatively larger rf trapping loss than the optimized setting in a real machine. Figure 12 shows the distributions in the phase spaces at the injection point and after some turns with an anticorrelated beam.

Consistent to the studies with ring-type distributions, the overall simulations with more or less realistic longitudinal motion show good momentum collimation efficiency with an anticorrelated distribution but much worse with a correlated distribution. As revealed by the simulations with a ring type of distribution, for a correlated beam, most uncontrolled particle losses mainly occur at the quadruples in the middle arcs where dispersion is large. The collimation efficiencies under different conditions are summarized in Table VI. Different transverse collimation efficiencies (100\% and $95 \%$ ) are used for the comparison. It also shows the simulation results with higher injection energies of 130

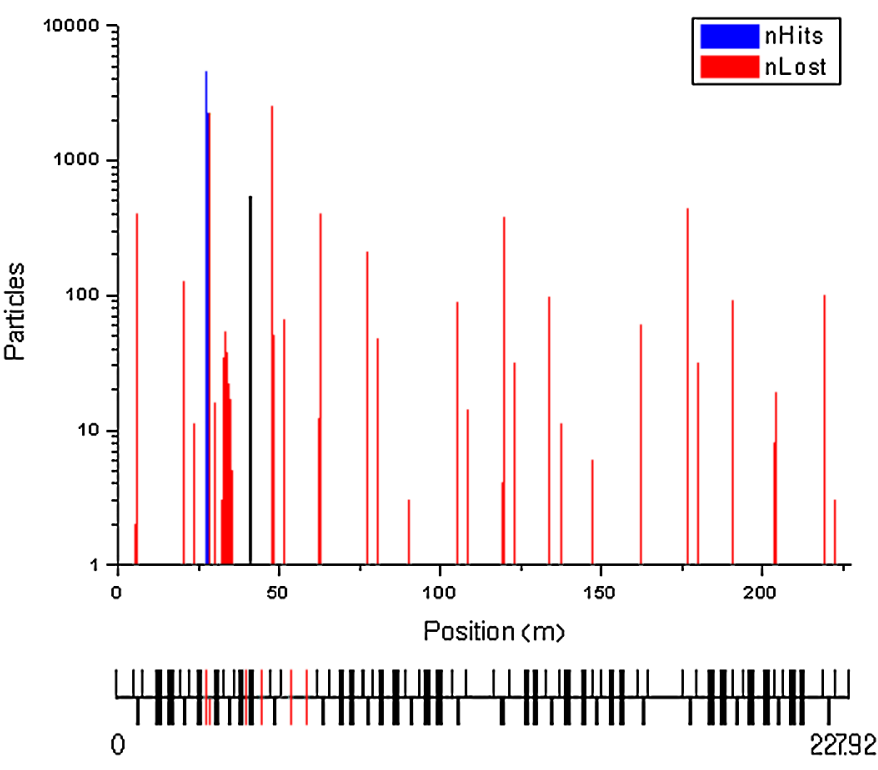

FIG. 13. Distribution of the lost particles along the ring and the hitting particles at the collimators in the CSNS RCS. nHits denotes the number of particles hitting on the collimators and nLost denotes the number of the absorbed particles. A total of 50000 particles are injected. The simulations cover the energy range of 80-90 MeV corresponding to CSNS-I phase. Left: anticorrelated beam; right: correlated beam. 
and $250 \mathrm{MeV}$. After the thicknesses of the primary and the secondary collimators adjusted to the beam energy, we can obtain almost similar momentum collimation efficiency with an anticorrelated beam, perhaps slightly worse with higher energy; however, the collimation efficiency with a correlated beam becomes much worse with increasing energy. Slightly higher collimation efficiency at $130 \mathrm{MeV}$ for the anticorrelated beam may be due that the loss mechanism or rf voltage pattern used in the simulations is more favorable for the momentum collimation. If a tertiary collimator in nickel or tungsten with a length of $0.5 \mathrm{~m}$ and a rectangular shape is placed in the same long drift of the middle arc, the collimation efficiency at $250 \mathrm{MeV}$ can be maintained almost the same as at $80 \mathrm{MeV}$. Figure 13 shows the distribution of the lost particles along the ring and the hitting particles at the collimators.

\section{CONCLUSIONS}

The simulation studies with coasting ring-type beams and more-or-less realistic beams show that the combined momentum collimation method is an effective method in compact synchrotrons of medium energy and high intensity. The particles with large positive momentum deviations can be effectively collimated by the combination of a thin carbon foil as the primary momentum collimator and the whole transverse collimators as the secondary collimation. The particles with large negative momentum deviations can be effectively collimated by the combination of a thin tantalum foil as the primary momentum collimator, the whole transverse collimators and a nickel block at the proximity of the primary momentum collimator together as the secondary collimators. The studies also show that the momentum collimation efficiency is much higher for an anticorrelated beam than for a correlated beam. This means that the injection with anticorrelated painting is favored. With higher injection energy, the collimation efficiency decreases slightly but can be compensated by adding a tertiary momentum collimator. Although the studies have been carried out with the CSNS parameters, the method should be applicable to other compact highintensity synchrotrons with available space at large dispersion region.

\section{ACKNOWLEDGMENTS}

This work was supported by the National Natural Science Foundation of China (10975150, 10775153), the CAS Knowledge Innovation Program-"CSNS R\&D Studies". The authors would like to thank CSNS colleagues for discussions.

\section{APPENDIX: DESIGN FEATURES AND MAIN PARAMETERS OF THE CSNS RCS}

CSNS will be constructed in three phases, $100 \mathrm{~kW}$ at CSNS-I, $200 \mathrm{~kW}$ at CSNS-II, and $500 \mathrm{~kW}$ at CSNS-III. The
TABLE VII. Main parameters of CSNS.

\begin{tabular}{lccc}
\hline \hline & CSNS-I & CSNS-II & CSNS-III \\
\hline Beam power $(\mathrm{kW})$ & 100 & 200 & 500 \\
Repetition rate $(\mathrm{Hz})$ & 25 & 25 & 25 \\
Average current $(\mu \mathrm{A})$ & 62.5 & 125 & 312.5 \\
Linac beam energy $(\mathrm{MeV})$ & 80 & 130 & 250 \\
Proton energy $(\mathrm{GeV})$ & 1.6 & 1.6 & 1.6 \\
RCS accumulated particles & $1.6 \times 10^{13}$ & $3.2 \times 10^{13}$ & $7.8 \times 10^{13}$ \\
\hline \hline
\end{tabular}

TABLE VIII. Main parameters of CSNS RCS.

\begin{tabular}{lc}
\hline \hline Circumference $(\mathrm{m})$ & 227.92 \\
Superperiod & 4 \\
Number of dipoles & 24 \\
Number of long drift & 12 \\
Total length of long drift $(\mathrm{m})$ & 75 \\
Betatron tunes $(\mathrm{h} / \mathrm{v})$ & $4.82 / 4.80$ \\
Chromaticity $(\mathrm{h} / \mathrm{v})$ & $-4.3 /-8.2$ \\
Momentum compaction & 0.041 \\
rf harmonics & 2 \\
rf frequency range $(\mathrm{MHz})$ & $1.0241-2.3723$ \\
rf voltage $(\mathrm{kV})$ & 165 \\
Transverse acceptance $(\pi \mathrm{mm}$ mrad $)$ & 540 \\
\hline \hline
\end{tabular}

main parameters are shown in Tables VII and VIII. The CSNS accelerator mainly consists of an $H^{-}$linac and a proton rapid cycling synchrotron (RCS). The upgrading will be achieved mainly by increasing the linac energy and accumulating more particles in the RCS.

As a high beam power synchrotron, the RCS has the design features such as large physical acceptance, fast acceleration and low beam loss, etc. A lattice of all triplet cells has been chosen as it can easily provide many uninterrupted long drifts required for the accommodation of $\mathrm{rf}$ stations, injection system, extraction system and collimation system, and reduce the size of the dipole magnets by defining small apertures. Space charge effects are key in operating the high-intensity accelerator, and higher injection energy is required to alleviate them at the upgrading phases. For the hands-on maintenance of the accelerator, a collimation system of good efficiency has been designed to localize beam losses.

[1] J. B. Jeanneret, Phys. Rev. ST Accel. Beams 1, 081001 (1998).

[2] C.M. Warsop, in the 29th ICFA Advanced Beam Dynamics Workshop on Beam Halo Dynamics, Diagnostics, and Collimation (HALO '03), Montauk, New York, 2003 (Springer, New York, 2003), pp. 154-157.

[3] K. Yamamoto, Phys. Rev. ST Accel. Beams 11, 123501 (2008). 
[4] U. Rohrer, PSI Graphic Turtle Framework based on a CERN-SLAC-FERMILAB version by K.L. Brown et al., 2006.

[5] J.D. Galambos et al., ORBIT User Manual Version 1.10, 1999.

[6] S. X. Fang, S. N. Fu, Q. Qin, J. Y. Tang, S. Wang, J. Wei, and C. Zhang, J. Korean Phys. Soc. 48, 697 (2006) [http://www.kps.or.kr/jkps/abstract_view.asp?articleuid= BD613E79-3430-4AC1-9637-063FC56AF2DF\& globalmenu=3\&localmenu=10].

[7] J. Y. Tang, S. N. Fu, and L. Ma, in Proceedings of the 46th ICFA Advanced Beam Dynamics Workshop on HighIntensity-High-Brightness Hadron Beams, 2010, Morschach, Switzerland [http://accelconf.web.cern.ch/ AccelConf/HB2010/].

[8] R. A $\beta$ mann et al., in Proceedings of EPAC 2004 (EPSAG, Lucerne, 2004), pp. 536-538 [http://accelconf.web .cern.ch/AccelConf/e04/].

[9] R. A $\beta$ mann et al., in Proceedings of the 46th ICFA Advanced Beam Dynamics Workshop on HighIntensity-High-Brightness Hadron Beams, 2010, Morschach, Switzerland [http://accelconf.web.cern.ch/ AccelConf/HB2010/].
[10] D.E. Johnson, in Proceedings of the 2007 Particle Accelerator Conference, Albuquerque, New Mexico (IEEE, New York, 2007), p. 1967.

[11] J. Y. Tang, J. F. Chen, and Y. Zou, in Proceedings of the 46th ICFA Advanced Beam Dynamics Workshop on HighIntensity-High-Brightness Hadron Beams, 2010, Morschach, Switzerland [http://accelconf.web.cern.ch/ AccelConf/HB2010/].

[12] J. Wei, Rev. Mod. Phys. 75, 1383 (2003).

[13] G. Moliere, Z. Naturforsch. 3a, 78 (1948) [http:// www.znaturforsch.com/aa/v03a/c03a.htm].

[14] P. J. Bryant and E. Klein, CERN Report No. SL/92-40, 1992.

[15] N. Catalan-Lasheras, in Proceedings of the 2001 Particle Accelerator Conference (IEEE, Piscataway, NJ, 2001), p. 1520 .

[16] J.F. Ziegler, M.D. Ziegler, J.P. Biersack, SRIM, 2006.

[17] N. Wang, N. Huang, Q. Qin, and S. Wang, in Proceedings of the 46th ICFA Advanced Beam Dynamics Workshop on High-Intensity-High-Brightness Hadron Beams, 2010, Morschach, Switzerland [http://accelconf.web.cern.ch/ AccelConf/HB2010/]. 